\title{
Development and Validation of a Questionnaire to Evaluate Medical Students' Evidence-Based Medicine Competencies
}

\author{
Jaehyun Park', Taeyoung Yoon ${ }^{2}$ and Jinkyung Ko ${ }^{1}$ \\ Departments of ${ }^{1}$ Medical Education, and ${ }^{2}$ Preventive Medicine, Kyung Hee University School of Medicine, \\ Seoul, Korea
}

\section{의대생의 근거중심의학(Evidence-Based Medicine) 수행역량 평가를 위한 질문지 개발 및 타당화}

경희대학교 의학전문대학원 ${ }^{1}$ 의학교육학교실, ${ }^{2}$ 예방의학교실

박재현 ${ }^{1}$, 윤태영 ${ }^{2}$, 고진경 ${ }^{1}$

Purpose: The purpose of the study was to develop and validate a questionnaire to evaluate medical students' knowledge of, attitude towards and practice of evidence-based medicine (EBM).

Methods: The participants of the study were 418 medical students enrolled in the Kyung Hee University School of Medicine in Seoul, Korea. To examine construct validity of the questionnaire, an exploratory factor analysis (EFA) was performed with 118 participants; a confirmatory factor analysis (CFA) was conducted with the remaining 281 participants. We developed 41 items with a 4-point Likert scale. An EFA was performed to verify the emergence of four dimensions of EBM competencies. The principal axis factoring method and the direct oblimin rotation method were used. To confirm construct validity, a CFA was conducted with the remaining 281 participants. To evaluate model fitness, root mean square error of approximation (RMSEA) and comparative fit index (CFI) were used as fit indices. We conducted ANOVA with Scheffe as discriminant validation, and calculated Cronbach's $\alpha$ of 4 subscales as reliability checkup.

Results: After refinement procedure, factor analysis of the 32 items in therevised questionnaire yielded 4 factors. The Scree plot supported a 4 factor solution explaining $53.5 \%$ of the variance. The 4 components derived were: factor 1 knowledge on EBM (11 items; Cronbach's $\alpha=0.92)$; factor 2_ pursuit towards EBM (10; 0.88); factor 3_reluctance on EBM (7; 0.78); factor 4 _practice of EBM $(4 ; 0.75)$. The questionnaire could discriminate competence differences among 1-3 yr students. Satisfactory Cronbach's $\alpha$ scores were noted for each factor as well.

Conclusion: The EBM competency questionnaire was validated.

Key Words: Evidence-based medicine, Undergraduate medical education, Educational measurement

Received: March 10, 2009 - Accepted: June 10, 2009 Corresponding Author: Jinkyung Ko

Department of Medical Education, Kyung Hee University School of Medicine, 1 Hoegi-dong, Dongdaemun-gu, Seoul 130-701, Korea

TEL) 02-961-9102 FAX) 02-969-0792 E-mail) michkay@khu.ac.kr

*This work was presented as a poster at the AMEE 2008 Conference in Prague.
Korean J Med Educ 2009 Sep; 21(3): 259-267. doi: 10.3946/kjme.2009.21.3.259.

(C) The Korean Society of Medical Education. All rights reserved. 


\section{서론}

근거중심의학(evidence-based medicine, EBM) 수행능 력은 이제 의사들이 갖추어야 할 핵심역량의 하나가 되었다 [1]. 1992년에 EBM의 개념과 필요성이 소개된 이후[2], 북미 와 유럽의 많은 의과대학은 EBM 수행에 필요한 기술을 훈련 하기 위한 교육프로그램을 적극적으로 도입했고, 교육의 긍 정적인 결과를 발표해 왔다[3,4,5,6,7]. 보고된 대부분의 훈 련 프로그램은 $\mathrm{EBM}$ 수행에 필요한 지식과 기술습득에 초점 이 맞추어져 있다. 그러나 많은 임상의들이 단기 EBM 훈련 프로그램을 통해 필요한 자료를 검색하고, 통계를 포함한 자 료의 내용을 이해하는 인지적인 능력을 습득했음에도, 이러 한 능력들이 실제 임상문제를 다루는 데 직접적으로 활용되 지 못했으며, 임상적 의사결정에서 자신감을 높여주지 못했 다[8,9].

이와 같이 많은 $\mathrm{EBM}$ 훈련 프로그램에서 학생과 의사들이 높은 성취도를 보임에도 불구하고, 임상현장에서의 실제적인 $\mathrm{EBM}$ 수행은 쉽게 향상되지 않는다. 그 첫 번째 이유로 훈련 프로그램의 내용을 지적할 수 있다. 많은 EBM 훈련프로그램 은 근거를 검색하고, 이를 비판적으로 판단하는 등과 같이 세 분화된 인지적 기술습득에 편중되어 있다[3,4]. 그러나 이러 한 인지적 기술의 부족이 의대생이나 수련의들의 EBM 수행 을 저해하는 가장 중요한 요소는 아니다. 연구에 의하면, 이들 이 임상현장에서 $\mathrm{EBM}$ 수행을 하기 어려운 이유는 $\mathrm{EBM}$ 의 필요성에 대한 인식이 부족하고, $\mathrm{EBM}$ 접근이 필요한 상황임 을 간파해서 시의적절하고 효율적으로 배운 기술을 적용하는 능력이 부족하며[10], 임상현장에서 임상교수들이 EBM 수행 을 지원하지 않고[7,11], 또한 시간적 제약[10]을 많이 받는 등 인식과 태도 그리고 실천의 측면에서 더 많이 찾아볼 수 있다[12].

$\mathrm{EBM}$ 훈련과 수행 간 괴리를 설명할 수 있는 두 번째 이유 로는 훈련프로그램의 결과를 평가하는 방법과 도구의 문제를 들 수 있다. EBM 교육의 평가도구에 대하여 체계적으로 분 석한(systemic review) 메타연구에 의하면, 보고된 $\mathrm{EBM}$ 수 행능력을 훈련하는 프로그램의 긍정적인 결과가 실제로는 본 질적으로 편향된 평가 설계로부터 얻어진 것들이 많았다[13].
더구나 적절한 타당화 과정을 거쳐 개발된 도구를 사용한 연 구는 거의 없었고, 몇몇 평가도구의 타당화 과정을 언급한 연 구들도 세부적인 타당화 방법을 보고하지 않았다. 이러한 훈 련 프로그램 결과평가의 신뢰성과 타당성에 대한 우려가 본 격화되면서 평가방법과 평가도구의 신뢰도와 타당도를 높이 는 것이 $\mathrm{EBM}$ 훈련 분야의 주요 쟁점으로 논의되기 시작하였 다[14].

지금까지 기술한 상황은 EBM 훈련을 일찍 시작한 유럽과 북미의 실정이다. 국내 $\mathrm{EBM}$ 훈련의 현실은 이러한 수준에 미치지 못한다. 의과대학 정규교육과정에서 $\mathrm{EBM}$ 을 훈련하 고 있는 대학은 국내에는 거의 없으며, EBM 훈련을 위한 단 기과정도 찾아보기 힘들다. 전공의 수련과정에서 컨퍼런스나 저널클럽 등의 형식으로 부분적으로 이루어지거나, 의대생 실습과정에서 간접적으로 경험할 수 있을 뿐이다[15]. 많은 임상의사나 교수들 중 $\mathrm{EBM}$ 수행에 대한 개념이 모호하거나 훈련 필요성에 대해 부정적인 시각을 가지고 있는 경우도 적 지 않다. 이러한 현실을 반영하듯 국내에서 체계적인 EBM 훈련 프로그램을 시행하고 그 결과를 보고한 논문의 수는 매 우 적으며, 결국 의학교육 분야에서 $\mathrm{EBM}$ 수행능력 훈련에 대한 본격적인 논의가 아직 시작되지 않았다고 할 수 있다. 이 에 연구팀은 체계적인 $\mathrm{EBM}$ 훈련 프로그램을 기획하기 위한 프로젝트에 착수하였다. 국내 의대생들에게 적합한 $\mathrm{EBM}$ 훈 련의 내용과 방법에 대한 일련의 연구 중 하나로 의대생들의 $\mathrm{EBM}$ 수행능력과 태도를 평가하는 도구를 개발하고 타당화 하였다.

이 연구의 목적은 의대생이 EBM 수행에 대한 지식, 태도, 실천을 평가할 수 있는 질문지를 개발하고, 타당화 하는 것이 다. 연구를 통해 개발한 질문지는 인지적 측면뿐 아니라 태도 와 실천을 포함하고 있어 포괄적인 평가가 가능하다. 또한 다 면적인 타당화 작업을 통해 도구의 타당성과 신뢰도를 높이 고자 하였으며, $\mathrm{EBM}$ 수행의 초심자에 해당하며 상대적으로 연구가 부족한 의대생(undergraduate medical education, $\mathrm{UME})$ 을 대상으로 개발하였다. 


\section{대상 및 방법}

\section{1. 대상}

이 연구는 서울 소재 경희대학교 의학전문대학원 학생 418 명을 대상으로 실시하였다. 경희대학교 의학전문대학원은 교 육과정에 $\mathrm{EBM}$ 훈련을 위한 명시적 교과목을 가지고 있지는 않다. 그러나 학생들은 2학년 PBL 과정과 3, 4학년 임상실습 과정을 통해 $\mathrm{EBM}$ 실행에 필요한 지식과 기술을 습득하고, 내재된 형태의 EBM 실행훈련을 받는다.

연구는 불성실한 응답을 제외하고 총 399명의 자료를 분석 했으며, 분석 자료의 학년별 구성은 1학년 108명, 2 학년 84명, 3 학년 89명, 그리고 4학년 118명으로 되어 있다. 이 중 4학년 은 탐색적 요인분석을 위한 1차 자료수집 대상이었고, 1 3학 년은 확인적 요인분석을 위한 2차 자료수집 대상이었다. 2차 자료수집에는 탐색적 요인분석 결과에 따라 수정된 질문지를 사용하였으며, 변별타당화에도 2차 수집 자료가 활용되었다. 자료 수집은 2007년 10월부터 2008년 2월까지 진행되었다.

\section{2. 측정도구}

질문지의 개발은 문헌분석과 1 차 질문지의 탐색적 요인분 석, 그리고 2 차 질문지의 타당화 등 3 단계 과정을 통해 진행 되었다.

먼저, 문헌분석은 PubMed를 이용하여 1998년부터 2007년 사이에 발표된 논문 중 'evidence-based medicine’, 'EBM training', 'undergraduate medical education', and 'educational measurement' 등의 키워드로 검색된 논문 10 편을 대상으로 하였다 $[5,16,17,18,19,20,21,22,23,24]$. 검색된 논문은 의대생들의 $\mathrm{EBM}$ 수행에 필요한 지식과 기술, $\mathrm{EBM}$ 에 대한 태도, 그리고 $\mathrm{EBM}$ 의 실천에 관한 항목들을 포함하 였다. 이에 따라 $\mathrm{EBM}$ 에 관한 지식, 태도, 실천을 기본 구조로 질문지 초안을 개발하였으며 각 하위구조에 포함되는 문항들 은 선행연구의 질문지를 참조하여 구체화하고[13], 국내 의대 의 $\mathrm{EBM}$ 훈련 상황을 고려하여 구성하였다. 개발된 예비설문 지는 의학교육담당교수와 임상실습담당교수의 검증을 통해 내용 타당도를 확인하였고, 4학년 학생 5 명에게 예비검사를
실시하여, 각 문항에 대한 이해도를 확인하여 1차 질문지를 확정하였다.

1 차 질문지는 $\mathrm{EBM}$ 에 관한 지식, 태도, 그리고 실천 정도를 측정할 수 있는 41 개 문항들로 구성하였으며, 각 문항은 Likert 4점 척도로 자신의 생각이나 행동에 일치하는 정도를 ‘전혀 그렇지 않다(1점)'에서 '매우 그렇다(4점)' 사이에 기록 하도록 되어 있었다.

1 차 질문지의 탐색적 요인분석 결과에 따라 4 개 요인이 추 출되었고, 9 개 문항이 제거되어 32문항의 2차 질문지로 수정 되었다. 수정된 질문지는 확인적 요인분석과 타당화를 통해 측정도구로서의 적합성을 평가하였다.

\section{3. 분석방법}

수집된 자료는 요인분석과 측정도구의 적합성 평가, 변별 타당도 확인을 위한 분산분석, 그리고 신뢰도 평가 등의 방법 으로 분석하였다. 요인분석에서는 주축요인추출방법(principal axis factoring)을 사용하여 요인을 추출하였고, 측정도 구의 적합성 평가는 공변량행렬자료를 이용한 최대우도추정 법(maximum lkelihood estimation)을 사용하여 4개요인구 조모형의 적합성을 검증하였다. 변별타당도를 검증하기 위해 서 학년을 변인으로 하여 분산분석(ANOVA)하고 사후분석 (Scheffe) 하였으며, 신뢰도는 내적일관성 계수(Cronbach's $\alpha)$ 를 산출하여 확인하였다. 자료분석에는 SPSS 12.0 (SPSS Inc., Chicago, USA)과 AMOS 5.0 (SPSS Inc.)을 사용하였 다.

\section{결과}

\section{1. 탐색적 요인분석을 통한 질문지의 구인타당도 (construct validity) 검증}

먼저 수집된 자료가 요인분석을 하기에 적합한 자료인지 여부를 판단하기 위하여 Kaiser-Meyer-Olkin (KMO)와 Bartlett 검정을 실시하였다. Table 1에 따르면 $\mathrm{KMO}$ 표본적 합성측도(measure of sampling adequacy)는 1차 자료가 $0.81,2$ 차 자료가 0.86 이다. 이는 $\mathrm{KMO}$ 값이 1 에 가까울수록 
Table 1. KMO Measure of Sampling Adequacy \& Bartlett' Test of Sphericity

\begin{tabular}{lrr}
\hline & 1st data & 2nd data \\
\hline KM0 measure of sampling adequacy & 0.81 & 0.86 \\
Bartlett' test of sphericity & & \\
Approx. Chi-Square & 2481.49 & 4013.51 \\
df & 630 & 496 \\
p-value & $<0.01$ & $<0.01$ \\
\hline
\end{tabular}

KMO: Kaiser-Meyer-Olkin.

Table 2. Factor Structure of the EBM-CQ by Direct Oblimin Rotation

\begin{tabular}{|c|c|c|c|c|}
\hline & 1 & 2 & 3 & 4 \\
\hline Kn9 & 0.838 & & & \\
\hline Kn5 & 0.789 & & & \\
\hline Kn10 & 0.785 & & & \\
\hline Kn11 & 0.741 & & & \\
\hline Kn3 & 0.737 & & & \\
\hline Kn8 & 0.696 & & & \\
\hline Kn1 & 0.689 & & & \\
\hline Kn7 & 0.688 & & & \\
\hline Kn4 & 0.659 & & & \\
\hline Kn2 & 0.655 & & & \\
\hline Kn6 & 0.576 & & & \\
\hline $\begin{array}{l}\text { Pu1 } \\
\text { Pu2 }\end{array}$ & & -0.775 & & \\
\hline Pu3 & & $\begin{array}{l}-0.151 \\
-0.698\end{array}$ & & \\
\hline Pu6 & & -0.665 & & \\
\hline Pu14 & & -0.637 & & \\
\hline Pu15 & & -0.622 & & \\
\hline Pu9 & & -0.615 & & \\
\hline $\begin{array}{l}\text { Pu11 } \\
\text { Pu4 }\end{array}$ & & -0.613 & & \\
\hline $\begin{array}{l}\text { Pu4 } \\
\text { Pu7 }\end{array}$ & & $\begin{array}{l}-0.609 \\
-0.608\end{array}$ & & \\
\hline Re16 & & & 0.757 & \\
\hline Re17 & & & 0.688 & \\
\hline Re10 & & & 0.594 & \\
\hline Re12 & & & 0.592 & \\
\hline Re13 & & & 0.582 & \\
\hline Re8 & & & $\begin{array}{l}0.502 \\
0\end{array}$ & \\
\hline $\begin{array}{l}\text { Re5 } \\
\text { Pr9 }\end{array}$ & & & 0.343 & \\
\hline $\mathrm{Pr} 8$ & & & & -0.791 \\
\hline Pr4 & & & & -0.516 \\
\hline $\mathrm{Pr} 7$ & & & & -0.509 \\
\hline
\end{tabular}

표본의 상관이 요인분석을 하기에 적합하며, 0.80 이상이면 양호하다는 Kaiser의 준거에 따라 본 연구의 자료가 요인분 석에 적합한 자료임을 설명한다[25]. 또한 Bartlett 구형성 검 사결과도 1,2 차 자료 모두 변수들 간의 상관계수가 통계적으
Table 3. Correlation Matrix between Factors

\begin{tabular}{ccccc}
\hline Factor & 1 & 2 & 3 & 4 \\
\hline 1 & 1 & & & \\
2 & -0.12 & 1 & & \\
3 & 0.08 & 0.17 & 1 & \\
4 & -0.28 & 0.25 & -0.06 & 1 \\
\hline
\end{tabular}

로 유의하므로 자료의 적합성을 재확인할 수 있다.

Table 2는 2차 수집 자료를 요인 분석하여 산출한 요인구 조행렬이다. 요인추출방법으로 주축요인추출방법(principal axis factoring)을 사용하였으며, 적정 요인 수는 스크리 도표 (Scree plot)를 참조하고 해석가능성을 고려하여 결정하였다. 또한 요인의 해석을 용이하게 하기 위하여 요인 간 상관을 허 용하는 직접 오블리민(direct oblimin) 방법을 사용하여 요인 을 회전시켰다. 탐색적 요인분석결과 총 4 개 요인이 추출되었 고, 이의 설명변량은 $53.5 \%$ 이다. 추출된 요인구조행렬은 1 차 자료로 시행한 탐색적 요인분석에서 추출된 요인 및 구성문 항과 일치하였다.

연구를 통해 개발된 32문항의 EBM 질문지는 4개 요인을 포함한다. 요인 1은 EBM 실행에 필요한 지식에 관한 11개의 문항으로 구성되어 '지식(Knowledge)'으로 이름 붙였다. 요 인 2는 $\mathrm{EBM}$ 훈련과 수행에 적극적인 태도를 설명하는 10 개 문항을 포함하므로 ‘추구태도(Pursuit)’로, 요인 3은 EBM의 효과성과 필요성에 대한 의구심을 표현하는 7개 문항을 포함하 므로 '기피태도(Reluctance)'로 명하였다. 요인 4는 EBM의 구 체적인 실천을 설명하는 4개 문항을 포함하며 '실천(Practice)' 이라 명명하였다. 질문지의 구조와 문항은 Appendix로 첨부하 였다.

Table 3은 요인 간 상관행렬이다. 모든 요인에서 유의한 상 관 값이 나타나지 않았으므로 4 요인은 각기 다른 측면을 측 정하고 있다.

\section{2. 확인적 요인분석을 통한 질문지 구조의 적합성 (fitness of structural model) 평가}

질문지 구조의 적합성을 평가하기 위하여 탐색적 요인분석 에 사용되지 않은 1 3학년 281명의 자료를 가지고 확인적 요 인분석을 실시하였다. 확인적 요인분석을 통해 얻은 모형의 
주요 적합도 지수(fit index)는 Table 4와 같다. 구조방정식 모형분석 결과는 $\chi^{2}$ 통계량과 자유도(df), $\mathrm{p}$-value를 제시한 다. 일반적으로 $\mathrm{p}-\mathrm{value}$ 가 0.05 보다 크면 적합도가 높은 것 으로 해석하는데 $\mathrm{p}$-value는 $\chi^{2}$ 통계량이 작을수록 커지게 된다. 그런데 $\chi^{2}$ 통계량은 표본의 크기가 커질수록 커지며, 측정변수의 수가 많아지면 커질 가능성이 높아진다. 그러므 로 표본의 크기가 크고 측정변수가 많은 자료에서 $\mathrm{p}$-value가 0.05 보다 작게 나타나더라도 적합도가 낮다고 말할 수는 없으 며, 다른 지표들과 함께 적합도를 판단해야 한다[26]. 표본크 기에 영향을 받지 않는 적합도 지수를 살펴보면 root mean square error of approximation (RMSEA)이 있다. 이는 $\chi^{2}$ 값을 자유도와 표본의 크기로 조정한 값이다. 대체로 0.08 보 다 작으면 적합도가 좋다고 할 수 있으며, 특히 $90 \%$ 신뢰구간 에서 상한 값이 0.08 보다 낮으면 적합도가 상당히 높다고 할

Table 4. Fit Indices of the EBM-CO Structural Model

\begin{tabular}{lcccc}
\hline Fit index & $\chi^{2}$ & df & RMSEA (90\% CI) & CFI \\
\hline Model & $1146.539^{\text {a) }}$ & 458 & $0.073(0.068 \sim 0.079)$ & 0.825 \\
\hline
\end{tabular}

RMSEA: Root mean square error of approximation, Cl: Confidence interval, CFI: Comparative fit index.

${ }^{a)} p<0.01$.
수 있다[27]. 연구에서 분석한 모형의 RMSEA는 0.073 으로 $90 \%$ 신뢰구간에서 0.068 0.079를 나타내 높은 적합도를 보 였다. Comparative fit index (CFI)는 연구모형과 null모형 을 비교해서 산출한 지수로 그 값이 0 과 1 사이에 있으면서 0.90 이상이면 좋은 적합도를 보이는 것으로 간주한다[27]. 본 모형은 0.825 를 나타내 기준값에 근접한다.

\section{3. 변별타당화(discriminant validation)}

변별타당화를 위하여 1 3학년 학생들의 하위척도별 점수 를 비교하였다[12,14]. $\mathrm{K}$ 의학전문대학원은 학생들의 EBM 훈련을 교육과정에 공식적으로 도입하지는 않았으나, 2학년 의 $\mathrm{PBL}$ 과정과 3학년의 임상실습과정을 통해 $\mathrm{EBM}$ 실천에 필요한 지식이나 실행방법을 간접적으로 접할 수 있으므로 학생들은 학년이 높아질수록 $\mathrm{EBM}$ 의 지식이나 실천경험이 많아진다. 질문지가 이러한 대상 집단의 차이를 적절히 변별 하는지 확인하기 위하여 4 개 하위척도의 학년 간 평균을 비교 하였다.

Table 5에 따르면 EBM 지식, 추구태도, 실천 척도에서 학 년 간 유의미한 차이가 있었고, 기피태도는 학년 간 차이가 없 었다. 학년 간 유의미한 차이가 검증된 지식, 추구태도, 실천 등 3 개 하위척도의 학년별 평균값의 사후검정을 한 결과, 지

Table 5. Results of ANOVA \& Scheffe for Discriminant Validation

\begin{tabular}{|c|c|c|c|c|c|c|c|}
\hline \multirow{2}{*}{ Sub Scale } & \multirow{2}{*}{$\mathrm{Yr}$} & \multirow{2}{*}{$n$} & \multirow{2}{*}{ Mean (SD) } & \multirow{2}{*}{$\mathrm{F}$} & \multirow{2}{*}{$\mathrm{p}$-value } & \multicolumn{2}{|c|}{ Scheffe } \\
\hline & & & & & & SG 1 & SG 2 \\
\hline \multirow[t]{3}{*}{ Knowledge } & 1 & 108 & $21.59(7.23)$ & & & $A$ & \\
\hline & 2 & 84 & 26.24 (5.89) & $11.99^{\mathrm{al}}$ & 0.00 & & B \\
\hline & 3 & 89 & 24.78 (6.73) & & & & B \\
\hline \multirow[t]{3}{*}{ Pursuit } & 1 & & 29.82 (6.90) & & & $A$ & \\
\hline & 2 & & 30.30 (4.35) & $3.67^{\mathrm{al}}$ & 0.03 & $A$ & B \\
\hline & 3 & & 31.85 (3.96) & & & & $B$ \\
\hline \multirow[t]{3}{*}{ Reluctance } & 1 & & $15.03(3.43)$ & & & & \\
\hline & 2 & & $15.33(3.17)$ & 2.16 & 0.12 & - & - \\
\hline & 3 & & 14.34 (2.87) & & & & \\
\hline \multirow[t]{3}{*}{ Practice } & 1 & & 9.59 (3.15) & & & A & \\
\hline & 2 & & 9.42 (2.68) & $9.98^{\mathrm{al}}$ & 0.00 & A & \\
\hline & 3 & & $11.12(2.51)$ & & & & B \\
\hline
\end{tabular}

SD: Standard deviation, SG: Subgroup.

${ }^{\mathrm{a})} \mathrm{p}<0.05$. 
Jaehyun Park, et al: Development and Validation of a Questionnaire to Evaluate Medical Students' EBM Competencies

Table 6. Internal Consistency of the EBM-CQ

\begin{tabular}{lc}
\hline EBM-CO & Cronbach's $\alpha$ \\
\hline Knowledge (11) & 0.92 \\
Pursuit (10) & 0.88 \\
Reluctance (7) & 0.78 \\
Practice (4) & 0.75 \\
EBM-CO total (32) & 0.86 \\
\hline
\end{tabular}

식의 경우, 1 학년과 2, 3 학년이 각각 2 개의 하위집단으로 구 분되었으며 2, 3학년의 점수가 1학년보다 높았다. 추구태도의 경우에는 1 학년과 3 학년이 각각 2 개의 하위집단을 구성하고, 2학년은 양 집단에 나뉘어 속하는 것으로 나타났으며 3학년 이 1학년보다 점수가 높았다. 실천의 경우, 1,2 학년과 3학년 이 각각 2 개의 하위집단으로 구분되었고, 3학년이 1,2 학년보 다 높은 점수를 기록하였다. 즉, 질문지는 학년에 따른 학생집 단의 EBM 지식, 태도, 실천의 차이를 변별해 낼 수 있었다.

\section{4. 신뢰도 검증}

내적일관성 분석결과, 지식 11문항이 Cronbach's $\alpha=0.92$, 추구태도 10 문항이 Cronbach's $\alpha=0.88$, 기피태도 7문항이 Cronbach's $\alpha=0.78$, 그리고 실천 4문항이 Cronbach's $\alpha=$ 0.75 로 나타났다. EBM_CQ 32문항 전체에 대한 Cronbach's $\alpha=0.86$ 로 만족할 만한 신뢰도 수준을 보였다(Table 6).

\section{고찰}

변별타당화 결과에 따르면 4개 영역이 학년에 따른 차이를 변별하는 정도가 다르다. 이를 자세히 살펴보면, 지식과 실천 영역에서는 학년 간 차이가 명확하게 나타났으나, 추구태도 는 유의도 수준을 0.01 로 설정했을 경우 $\mathrm{p}$-value (0.03)가 영 가설을 기각해야 수준이며, 기피태도의 값은 학년 간 차이를 변별하지 못했다. 이는 질문지의 두 영역이 학생들의 차이를 충분히 변별하지 못함을 나타내는 것이다.

그러나 추구태도와 기피태도가 학생들의 '태도'를 측정하 는 영역임으로 생각해 볼 때 위와 같은 결과를 두 영역이 변별 력이 낮은 것으로 결론 내리기 전에 연구자는 다른 설명을 시
도해 보고자 한다. 학년이 높아지면서 EBM 수행을 더 많이 경험할수록 학생들의 지식은 분명 늘어날 것이다. 또한 임상 실습이 늘어나면서 $\mathrm{EBM}$ 실천에 대한 필요성이 높아짐에 따 라 $\mathrm{EBM}$ 수행을 실천하는 행동도 더 많아질 것이다. 그러나 태도는 이 두 영역과는 다르게 변화한다. 비공식적 훈련 상황 에서의 개인적인 경험과 $\mathrm{EBM}$ 에 대한 교수나 선배의 태도는 학생들의 태도에 강력한 영향을 미치며, 결국 학생들의 태도 가 어떤 방향으로 발전될지 단언하기 어렵다. 그러므로 연구 자는 기피태도와 추구태도의 결과는 태도영역 훈련의 특수성 과 낮은 성공률을 나타내는 결과일 수도 있음에 주목하였다. 두 영역의 변별력이 떨어진 결과는 문항의 수정, 보완을 필요 로 할 뿐 아니라 EBM에 대한 긍정적인 태도를 높이고 부정 적인 태도를 낮추는 교육 프로그램을 기획할 때 특별한 주의 를 요함을 환기시키기도 한다.

다음으로 검증한 구조모형의 적합성 지수를 살펴보면, 증 분적합도지수(incremental fit indices)에 해당하는 CFI는 적정 적합도 수준인 0.90 에 다소 못 미치는 0.825 를 나타냈다. 비록 적정 적합도 수준에 근접하고는 있으나 후속 연구를 통 해 보완되어야 할 수치이다. 그러나 절대적합도지수(absolute fit indices)에 해당하는 RMSEA는 높은 적합도를 보이고 있 으며, 특히 신뢰구간의 $90 \%$ 상한값이 0.79 를 나타내 RMSEA 지수상에서 매우 높은 적합도를 보이고 있어[2] 구조의 타당 성에 대해 긍정적으로 해석하는 데 무리가 없다.

이 연구의 제한점은 타당화를 위한 연구대상이 $\mathrm{K}$ 의학전문 대학원 1 개 기관의 학생들로 한정되어 있다는 점과 $\mathrm{K}$ 의학전 문대학원에 공식적인 $\mathrm{EBM}$ 훈련프로그램이 준비되어 있지 않아 특정 훈련프로그램의 효과를 평가하는 차원에서 질문지 를 활용하지 못하고, 비공식적이고 내재적인 $\mathrm{EBM}$ 훈련 효과 를 가정하고 학생들의 $\mathrm{EBM}$ 관련 지식, 태도, 실천 정도를 측 정한 점을 지적할 수 있다. 또한 학생들의 $\mathrm{EBM}$ 수행능력을 측정하는 기존의 측정도구가 없어 공인된 다른 척도와의 비 교를 통해 타당화를 하는 준거타당도 검증을 하지 못한 점도 아쉬운 점이다[28].

이러한 제한점에도 불구하고 이 연구는 4 가지 이상의 타당 화 과정을 통해 질문지의 타당도와 신뢰도를 다각도에서 확 인함으로써 질문지의 충실도를 높였으므로 연구목적을 충실 히 달성하였다. 또한 연구에서 개발하고 타당화한 질문지는 
박재현 외: 의대생의 근거중심의학(Evidence-Based Medicine) 수행역량 평가를 위한 질문지 개발 및 타당화

의대생들의 $\mathrm{EBM}$ 관련 지식과 태도, 실천 정도를 측정할 수 있는 신뢰할 만한 도구로 활용될 수 있을 뿐 아니라 질문지의 구조와 문항의 내용이 향후 의대생들을 위한 $\mathrm{EBM}$ 수행훈련 프로그램을 설계하고 개발하는 데 기초적인 틀로도 활용될 수 있다는 점도 연구의 의의로 논의할 수 있다.

이 연구는 의대생을 대상으로 초보적인 EBM 능력수준을 측정하기 위하여 개발되었다. 만약 의대생들이 EBM 수행에 대해서 준비된 훈련을 받고 계획된 실행경험을 하게 된다면 지금보다 특화된 지식과 복합적인 실행을 묻는 문항으로 그 들의 지식과 실행수준을 측정해야 할 필요가 생길 것이다. 또 한 의대생뿐 아니라 전공의나 전문의를 위한 $\mathrm{EBM}$ 질문지나 교육 프로그램의 개발요구도 점점 높아지고 있으므로 이를 위한 확장된 질문지의 개발도 필요하다 $[3,6,29,30]$. 그러므로 후속연구를 통해 보다 다양한 수준에 따른 질문지가 개발될 필요가 있다. 아울러 $\mathrm{EBM}$ 관련 역량을 평가하는 질문지의 개발과 타당화 연구에 이 연구가 방법과 내용의 측면에서 도 움을 줄 수 있으리라 기대한다.

\section{REFERENCES}

1. World Federation for Medical Education. World Summit on Medical Education. Proceedings. Edinburgh, 8-12 August 1993. Med Educ 1994; 28 Suppl 1: 1-3.

2. Straus SE. Evidence-based medicine: how to practice and teach EBM. 3rd ed. Edinburgh, USA: Elsevier, Churchill Livingstone; 2005. p 1-3.

3. Smith CA, Ganschow PS, Reilly BM, Evans AT, McNutt RA, Osei A, et al. Teaching residents evidence-based medicine skills: a controlled trial of effectiveness and assessment of durability. J Gen Intern Med 2000; 15: 710-715.

4. Ghali WA, Saitz R, Eskew AH, Gupta M, Quan H, Hershman WY. Successful teaching in evidence- based medicine. Med Educ 2000; 34: 18-22.

5. Thomas PA, Cofrancesco J Jr. Introduction of evidencebased medicine into an ambulatory clinical clerkship. J
Gen Intern Med 2001; 16: 244-249.

6. Green ML, Ellis PJ. Impact of an evidence-based medicine curriculum based on adult learning theory. J Gen Intern Med 1997; 12: 742-750.

7. McAlister FA, Graham I, Karr GW, Laupacis A. Evidence-based medicine and the practicing clinician. J Gen Intern Med 1999; 14: 236-242.

8. Barnett SH, Kaiser S, Morgan LK, Sullivant J, Siu A, Rose D, et al. An integrated program for evidence- based medicine in medical school. Mt Sinai J Med 2000; 67: $163-168$.

9. Poses RM. Money and mission? Addressing the barriers to evidence-based medicine. J Gen Intern Med 1999; 14: 262-264.

10. Sackett DL, Straus SE. Finding and applying evidence during clinical rounds: the "evidence cart". JAMA 1998; 280: 1336-1338

11. Sackett DL, Parkes J. Teaching critical appraisal: no quick fixes. CMA J 1998; 158: 203-204.

12. Johnston JM, Leung GM, Fielding R, Tin KY, Ho LM. The development and validation of a knowledge, attitude and behaviour questionnaire to assess undergraduate evidence-based practice teaching and learning. Med Educ 2003; 37: 992-1000.

13. Shaneyfelt T, Baum KD, Bell D, Feldstein D, Houston TK, Kaatz S, et al. Instruments for evaluating education in evidence-based practice: a systematic review. JAMA 2006; 296: 1116-1127.

14. Taylor R, Reeves B, Mears R, Keast J, Binns S, Ewings $\mathrm{P}$, et al. Development and validation of a questionnaire to evaluate the effectiveness of evidence-based practice teaching. Med Educ 2001; 35: 544-547.

15. Lee YM. 'EBM'eui kyoyookhakjuk whalyongsarye. Yonsei J Med Educ 2001; 3: 37-45.

16. Dorsch J, Aiyer MK, Gumidyala K, Meyer LE. Retention of EBM competencies. Med Ref Serv Q 2006; 25: 45-57. 17. Bradley P, Oterholt C, Herrin J, Nordheim L, Bjørndal 
A. Comparison of directed and self-directed learning in evidence-based medicine: a randomised controlled trial. Med Educ 2005; 39: 1027-1035.

18. Weberschock TB, Ginn TC, Reinhold J, Strametz R, Krug D, Bergold M, et al. Change in knowledge and skills of Year 3 undergraduates in evidence-based medicine seminars. Med Educ 2005; 39: 665-671.

19. Ismach RB. Teaching evidence-based medicine to medical students. Acad Emerg Med 2004; 11: e6-el0.

20. Dorsch JL, Aiyer MK, Meyer LE. Impact of an evidence-based medicine curriculum on medical students' attitudes and skills. J Med Libr Assoc 2004; 92: 397-406.

21. Bergus G, Vogelgesang S, Tansey J, Franklin E, Feld R. Appraising and applying evidence about a diagnostic test during a performance-based assessment. BMC Med Educ 2004; 4: 20.

22. Holloway R, Nesbit K, Bordley D, Noyes K. Teaching and evaluating first and second year medical students' practice of evidence-based medicine. Med Educ 2004; 38: $868-878$.

23. Hunt DP, Haidet P, Coverdale JH, Richards B. The effect of using team learning in an evidence-based medicine course for medical students. Teach Learn Med
2003; 15: 131-139.

24. Srinivasan M, Weiner M, Breitfeld PP, Brahmi F, Dickerson KL, Weiner G. Early introduction of an evidence-based medicine course to preclinical medical students. J Gen Intern Med 2002; 17: 58-65.

25. Kaiser HF. An index of factorial simplicity. Psychometrika 1974; 39: 31-36.

26. Joo YJ, Kim NY, Cho HK. Test development and verifying the validity and reliability for measuring a effectiveness of e-learning course in cyber university. J Korean Inf Educ 2008; 12: 109-120.

27. Lee HS, Rhim JH. Structural equation modeling with AMOS 6.0. Seoul, Korea: Bobmunsa; 2007. p 32-35.

28. Bae HY, Rhee MK. Development of negative emotion-induced eating motive scale (NEIEMS). Korean J Health Psychol 2004; 9: 187-201.

29. Bordley DR, Fagan M, Theige D. Evidence-based medicine: a powerful educational tool for clerkship education. Am J Med 1997; 102: 427-432.

30. Green ML. Evidence-based medicine training in graduate medical education: past, present and future. J Eval Clin Pract 2000; 6: 121-138. 
Appendix. EBM 수행역량평가를 위한 질문지의 요인별 문항

\begin{tabular}{|c|c|c|c|c|c|}
\hline $\mathrm{N}$ & 문 항 & \multicolumn{4}{|c|}{ 그렇다 - 아니다 } \\
\hline Kn1 & 검색한 논문들의 자료의 수집과 분석방법이 적절한지 판단할 수 있다. & 4 & 3 & 2 & 1 \\
\hline Kn2 & 연구논문을 읽을 때 그 논문의 결과가 믿을 만한 것인지 아닌지 판단할 수 있다. & 4 & 3 & 2 & 1 \\
\hline Kn3 & 탐색한 근거에 기반을 두고 환자의 진단과 치료에 대한 의사결정을 내릴 수 있다. & 4 & 3 & 2 & 1 \\
\hline Kn4 & $\begin{array}{l}\text { 내가 가지고 있는 문제에 해답이 될 만한 근거를 교과서에서 찾아야 할지, 연구논문에서 } \\
\text { 찾아야 할지 판단할 수 있다. }\end{array}$ & 4 & 3 & 2 & 1 \\
\hline Kn5 & 명확하지 않은 임상 상황을 접했을 때 필요한 해답을 얻을 수 있는 질문을 만들 수 있다. & 4 & 3 & 2 & 1 \\
\hline Kn6 & MEDLINE에서 효율적으로 문헌 검색을 할 수 있다. & 4 & 3 & 2 & 1 \\
\hline Kn7 & 발표된 임상연구 논문을 비판적으로 평가할 수 있다. & 4 & 3 & 2 & 1 \\
\hline Kn8 & 어려운 환자 사례를 보고 내가 모르는 것이 무엇인지 말할 수 있다. & 4 & 3 & 2 & 1 \\
\hline $\mathrm{Kng}$ & $\begin{array}{l}\text { 환자 진료 시 명확하지 않은 부분을 탐색이 가능한 적절한 임상적 문제로 전환시킬 수 } \\
\text { 있다. }\end{array}$ & 4 & 3 & 2 & 1 \\
\hline Kn10 & $\begin{array}{l}\text { 검색한 자료에서 얻은 추상적이거나 부분적인 정보를 환자에게 도움이 될 수 있도록 } \\
\text { 활용할 수 있다. }\end{array}$ & 4 & 3 & 2 & 1 \\
\hline Kn11 & 검색한 근거와 내가 다루고 있는 환자와의 관련성을 판단할 수 있다. & 4 & 3 & 2 & 1 \\
\hline Pu1 & EBM을 실천하는 것은 의대생에게 중요한 일이다. & 4 & 3 & 2 & 1 \\
\hline Pu2 & EBM은 임상현장에서 유용한 방법이다. & 4 & 3 & 2 & 1 \\
\hline Pu3 & EBM은 patient outcome을 향상시킨다. & 4 & 3 & 2 & 1 \\
\hline Pu4 & EBM은 임상적 노력의 비용효과를 높일 수 있다. & 4 & 3 & 2 & 1 \\
\hline Pu6 & 몇 년 후 의사가 되면 나에게 $\mathrm{EBM}$ 은 지금보다 더 중요해질 것이다. & 4 & 3 & 2 & 1 \\
\hline Pu7 & EBM 교육과정이 있다면 교육을 받고 싶다. & 4 & 3 & 2 & 1 \\
\hline Pu9 & 몇 년 후 의사가 되면 나는 $\mathrm{EBM}$ 을 더 자주 활용할 것이다. & 4 & 3 & 2 & 1 \\
\hline Pu11 & EBM은 임상에서의 의사결정에 도움을 준다. & 4 & 3 & 2 & 1 \\
\hline Pu14 & $\begin{array}{l}\mathrm{EBM} \text { 을 통해 내가 하려고 하는 처치가 효과적이라는 것에 대한 확실성을 증가시킬 수 } \\
\text { 있다. }\end{array}$ & 4 & 3 & 2 & 1 \\
\hline Pu15 & EBM은 의대/의전원 교육과정의 중요한 부분이 되어야 한다고 생각한다. & 4 & 3 & 2 & 1 \\
\hline Re5 & EBM을 옹호하는 사람들은 주로 임상의사보다는 기초의학자들이다. & 4 & 3 & 2 & 1 \\
\hline $\operatorname{Re} 8$ & 대부분의 의학영역에서 활용할 만한 근거는 많지 않다. & 4 & 3 & 2 & 1 \\
\hline Re10 & EBM은 임상적인 경험과 직관의 가치를 떨어뜨린다. & 4 & 3 & 2 & 1 \\
\hline Re12 & EBM은 일상적인 임상활동에서 실용적이지 않다. & & 3 & 2 & 1 \\
\hline Re13 & EBM은 병력청취와 신체진찰 기술의 중요성을 낮춘다. & 4 & 3 & 2 & 1 \\
\hline Re16 & EBM은 한 시대에 나타나는 유행 같은 것이므로 나는 이를 받아들일 필요를 느끼지 못한다. & 4 & 3 & 2 & 1 \\
\hline Re17 & $\begin{array}{l}\text { 의학은 통계가 아니라 사람과 환자에 관한 것이므로 일반적으로 의사들은 } \mathrm{EBM} \text { 을 실천해서 } \\
\text { 는 안 된다. }\end{array}$ & 4 & 3 & 2 & 1 \\
\hline Pr4 & 의학적 근거를 찾기 위해 자료를 자주 탐색한다. & 4 & 3 & 2 & 1 \\
\hline $\operatorname{Pr} 7$ & 학년이 올라갈수록 $\mathrm{EBM}$ 이 점차 습관화된 일상이 되어간다. & 4 & 3 & 2 & 1 \\
\hline Pr8 & $\begin{array}{l}\text { 실습에서 불명확한 지식을 보완하기 위해서 PubMed, Medline과 같은 의학 분야의 학술데이 } \\
\text { 터 베이스를 검색하였다. }\end{array}$ & 4 & 3 & 2 & 1 \\
\hline $\operatorname{Pr} 9$ & 의학적 근거를 찾기 위해 연구논문 원본을 검색한다. & 4 & 3 & 2 & 1 \\
\hline
\end{tabular}

